\title{
Mesenchymal stem cell-derived exosome as a nano weapon to target the COVID-19 pandemic
}

\author{
YASHVI SHARMA; SUCHI GUPTA; SUJATA MOHANTY* \\ Stem Cell Facility (DBT-Centre of Excellence for Stem Cell Research), All India Institute of Medical Sciences, New Delhi, 110029, India
}

Key words: SARS-CoV-2, Wharton's Jelly, COVID-19, Cell-free therapy, Exosomes, Immunomodulation

\begin{abstract}
In these times of despair when a nano-sized organism, the SARS-CoV-2, has rendered the human race helpless, made the global health status decline, and drowned the world economy, a ray of hope comes from another nano-sized particle, the exosome. The potential of mesenchymal stem cells has already been established in COVID-19; however, cell-based therapy has its risks. We thereby propose cell-free therapy using stem cells-derived exosomes to fight against COVID-19, as they can be a game-changer owing to their immunomodulatory nature, which combats the cytokine storm characterizing this disease, and their practical efficiency, which will realistically aid large access to therapy worldwide.
\end{abstract}

\section{Introduction}

COVID-19 is a deadly disease that has caused worldwide havoc and shaken the core of the economy, even for the most developed countries. Moreover, the face of survival against COVID-19 has been divergent for people that belong to different socio-economic classes as the availability of the best healthcare services is significantly diminished to the impoverished part of the society, including developing countries and nations. Conditions are specifically severe in such regions due to factors like overpopulation, limited resources, logistic gaps, improper infrastructure, and poverty. Although the governments are taking several measures to ensure the safety of the citizens, the feasibility of medicine-for-all and medicine-to-all seems far-fetched considering the current scenario of therapy.

A therapeutic agent suitable to combat such a pathologically and socially complex situation needs to be physiologically efficient, mechanically flexible, and practical to use for feasibility in mass manufacturing. Considering all these properties, we can state that the therapeutic agent that displays these attributes is the exosome. These are small vesicles in the size range of $30-150 \mathrm{~nm}$, which are synthesized in the cell inside multivesicular bodies (MVBs) as intraluminal vesicles (ILVs) by double invagination of the plasma membrane. These are secreted upon the fusion of

*Address correspondence to: Sujata Mohanty,

drmohantysujata@aiims.edu

Received: 13 October 2020; Accepted: 21 December 2020
MVBs with the plasma membrane. Given their versatile properties and small size, exosomes offer novel opportunities for cell-free therapeutics and are state-of-art in regenerative medicine (Jabbari et al., 2020; Jing et al., 2018). The exosomes have proven potential as drug delivery carriers, and their immunomodulatory properties are unmatched, so employing them as a therapeutic agent against COVID-19 is an ideal approach (Akbari and Rezaie, 2020; Popowski et al., 2020). Among all the therapies tried, a major focus for the treatment of this disease has been shifted towards Mesenchymal Stem Cells (MSCs) and their secretome (Akbari and Rezaie, 2020). MSCs, apart from transdifferentiation, also actively exhibit their functions via the mechanism of paracrine signaling aided by the secreted extracellular vesicles, especially exosomes. Several clinical trials have employed MSCs and the exosomes secreted by them against the pathophysiology of COVID-19 and shown outstanding outcomes (Sengupta et al., 2020). Exosomes carry many bioactive molecules involved intricately in cell signaling and communication, and so we could infer that exosomes are reflected as a shadow of their parent cells per se and the flag holders of MSCs mechanism of action (Magdy Beshbishy et al., 2020).

Even though the regenerative potential of exosomes derived from MSCs has been explored widely, there are yet several roadblocks that hinder the commercialization of these nano-sized vesicles as a cell-free therapeutic agent. The primary concern lies in source selection. We would like to highlight that even though MSCs from all sources manifest similar properties, some minuscule differences are observed 
in their functioning depending upon their source, which therefore also confer differences to exosomes shed by them. In our view, the common sources of MSCs isolation like bone marrow, adipose tissue, dental pulp, etc., are immunologically unbenefited and hold a higher risk of packaging coronavirus or any other pre-existing infection into their exosomes, whereas Wharton's jelly derived MSCs (WJ-MSCs) are considered to be immunologically privileged due to the sheltered nature of their source, and because it is known to consists of exceedingly naive cells that are least exposed to environmental stresses (Marino et al., 2019; Bullard et al., 2019; Kamal and Kassem, 2020). WJ-MSCs are known to exhibit high immunosuppression, a property direly needed to fight COVID-19. This suggests that exosomes derived from WJ-MSC represent a better ability to manage this disease, given that SARS-CoV-2 bind to the ACE-2 receptors present on the ciliated secretory cells in the nasal epithelium with high affinity, forth which they replicate and propagate towards lungs where they enter using the ACE-2 receptors on type II pulmonary alveolar epithelial cells (Hassanpour et al., 2020; Parasher, 2020). This small virus then works by inducing a tempest of cytokines in the lungs by inciting some major proinflammatory factors like IL-2, IL-6, IL-7, and TNFa and activating inflammatory pathways including NF-AT, IRF-3, IRF-7, NF-kB, ATF-2/jun, and jun/fos (AP-1) (Emameh et al., 2020; Tay et al., 2020). This cascade attracts immune cells like monocytes, macrophages, and $\mathrm{T}$ cells, thereby leading to the creation of a cycle that increasingly intensifies inflammation (Shetty, 2020). Apart from many other probable causes, this loop of altered immunomodulation instigates some of the signature symptoms characterizing SARS-CoV-2, thereby causing lethal damages to the lung ultrastructure and multiple organs upon the propagation of this cytokine storm. Breaking into an immunologically complex milieu like this, while specifically targeting the virus, is an exasperating task. In such a conflicting situation, the instinctive immunosuppressive abilities of WJ-MSC-exosomes make them a trustable candidate.

MSC-derived exosomes are able to act against the pathophysiology of SARS-Cov-2 by virtue of their cargo. These nano-vesicles act as bullets encapsulating bioactive molecules including mRNAs, proteins, miRNAs, etc., which target the infected cells, thereby initiating an antiinflammatory and antiviral response, thus providing a reparative effect (Wang et al., 2019). miRNAs have been found to be the primal component to be transferred from exosomes to the 'cells-in-need', which could form the basis of another mechanism that could potentially be responsible for suppressing the proliferation of the virus via silencing action and epigenetic alterations (Gupta et al., 2020). MSCderived exosomes are immunomodulatory in nature as they down-regulate the pro-inflammatory cytokines including TNF- $\alpha$ and IFN- $\gamma$ which further subdues T-cell maturity while elevating the levels of anti-inflammatory cytokines like nitric oxide, TGF- $\beta$, and IL-10 (Xie et al., 2020). They have also been seen to modulate the function of B-cells by differentially regulating the expression of relevant gene encoding mRNAs (Khare et al., 2018).
Cell-free therapy using exosomes is considered safer than cell-based therapy using MSCs as exosomes cannot replicate, and therefore negate the risk of teratoma; even though multiple studies have proven otherwise, it remains a major concern pertaining stem cell transplantation (Jing et al., 2018; Abraham and Krasnodembskaya, 2020). Also, MSCs are large in size and are unable to cross physiological barriers, so they also hold a risk of blocking circulation and getting caught in lung vasculature, whereas exosomes have the ability to cross physiological barriers, are stable in circulation, and are non-toxic (Jing et al., 2018; Bang and Kim, 2019). They even display a lower risk of immune rejection when administered from an allogenic source (Chase and Gallicchio, 2019). The research to assess the capability of exosomes derived from MSCs to act as a drugdelivery vehicle by exosome engineering and manipulation of their surface and cargo is still in its infancy, but there have been several studies that prove its targeting ability and specificity in therapeutics (Choi et al., 2020; Guo et al., 2019). We would also like to add here that the viability of exosomes is unquestionable as 'live or dead' status cannot be assigned to them; however, we cannot assure that all their parent cells remain targeted and viable in vivo upon transplantation, hence making these nanoparticles a better therapeutic candidate than their parent cells itself.

Apart from their immensely elaborate therapeutic cascade, the undemanding operational protocols of exosomes highlight them as a practical candidate for offthe-shelf remedial approach against COVID-19, especially in developing countries, as exosomes can be easily procured, lyophilized, manufactured, and transported in different formulations like freeze-dried, sprays, ointment or injectables. These diverse features of the exosomes make them an appropriate nano-platform for future commercialization and biomedical applications. Certain startups like Exomedx, Codiak Biosciences, Exocyte Therapeutics, etc., have explored the commercialization of exosomes for regenerative medicine, e.g., Kimera labs have created an exosome-based product called $\mathrm{XoGlo}^{\mathrm{Tm}}$ for skin rejuvenation (Yousef and Abdelnaser, 2019). This will decrease the cost of therapy drastically, resolving the question of affordability and providing medicine-for-all, while the easy handling procedures will aid in logistics hence providing medical-to-all (Fig. 1). Few minor glitches that remain to be resolved like standardization in protocols, batch variations, and improving technological scalability, if circumvented, will highlight these nanovesicles as the key-players in the pharmaceutical industry (Jing et al., 2018; Bari et al., 2019).

Thus, we recapitulate from our article that using exosomes derived from MSCs as a cell-free therapeutic agent is a quintessential approach to combat COVID- 19. Exosomes derived from WJ-MSCs especially possess astonishing remedial capabilities, and their viability in vivo is explicitly assured. These natural nanoparticles can be maneuvered into different kinds of formulations as complex as vaccines or in commonly benchside therapeutics like nasal-sprays. Hence, the diverse scope and possibility of manipulations offered by MSC-derived exosomes make them an ultimate curative cell-free bioagent to defeat COVID-19. 


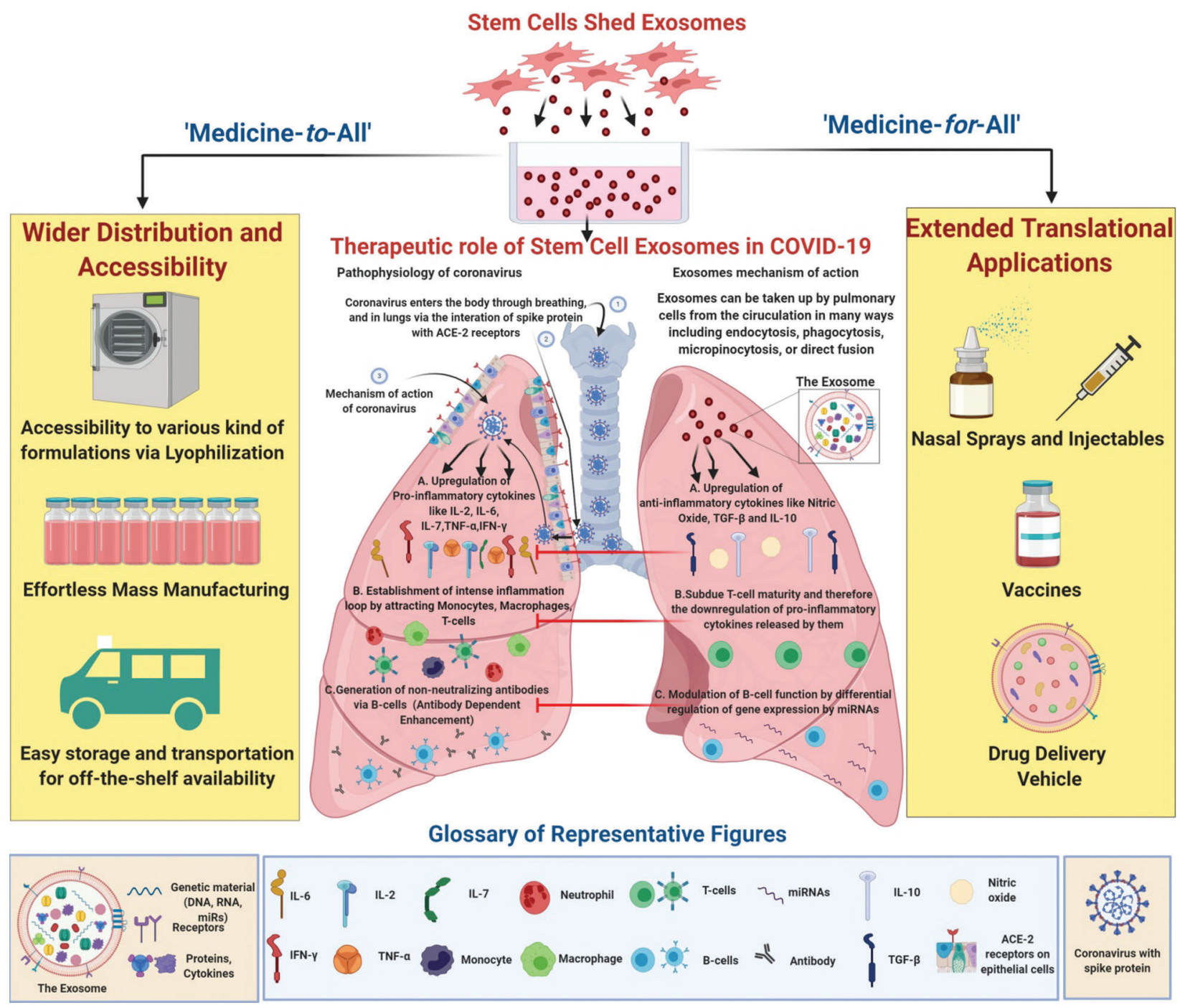

FIGURE 1. Stem Cell Exosomes as a bioagent to defeat COVID-19. Stem cell exosomes play a therapeutic role against the pathophysiology of COVID-19, majorly by targeting the pro-inflammatory cascade triggered by the coronavirus. Their practicality and diversity make them a potential front-line model to combat this pandemic by providing 'medicine-to-all', as their flexible properties aid in logistical purposes, while also providing 'medicine-for-all', as they can be manipulated into various therapeutic formulations.

Acknowledgement: The image was created using BioRender.com.

Author Contribution: The authors confirm contribution to the paper as follows: YS contributed to conceptualization, writing and graphics. SG contributed to critical evaluation of the manuscript. SM contributed to conceptualization, critical evaluation, and coordinated throughout the manuscript writing. All authors approved the final version of the manuscript.

Funding Statement: The author(s) received no specific funding for this study.

Conflicts of Interest: All authors have read the journal's policy on disclosure of potential conflicts of interest and have none to declare.

\section{References}

Abraham A, Krasnodembskaya A (2020). Mesenchymal stem cellderived extracellular vesicles for the treatment of acute respiratory distress syndrome. Stem Cells Translational Medicine 9: 28-38. DOI 10.1002/sctm.19-0205.
Akbari A, Rezaie J (2020). Potential therapeutic application of mesenchymal stem cell-derived exosomes in SARS-CoV-2 pneumonia. Stem Cell Research \& Therapy 11: 356. DOI 10.1186/s13287-020-01866-6.

Bang OY, Kim EH (2019). Mesenchymal stem cell-derived extracellular vesicle therapy for stroke: challenges and progress. Frontiers in Neurology 10: 211. DOI 10.3389/ fneur.2019.00211.

Bari E, Ferrarotti I, Torre ML, Corsico AG, Perteghella S (2019). Mesenchymal stem/stromal cell secretome for lung regeneration: the long way through "pharmaceuticalization" for the best formulation. Journal of Controlled Release 309: 11-24. DOI 10.1016/j.jconrel.2019.07.022.

Bullard JD, Lei J, Lim JJ, Massee M, Fallon AM, Koob TJ (2018). Evaluation of dehydrated human umbilical cord biological properties for wound care and soft tissue healing. Journal of Biomedical Materials Research Part B: Applied Biomaterials 107: 1035-1046. DOI 10.1002/jbm.b.34196.

Chase DM, Gallicchio VS (2019). The effect of mesenchymal stem cells and exosomes to treat idiopathic pulmonary fibrosis. Journal of Stem Cells Research and Regenerative Medicine 3: 1-10. 
Choi H, Kim Y, Mirzaaghasi A, Heo J, Kim Y N, Shin J H, Kim S, Kim $\mathrm{N} \mathrm{H}$, Cho E S, In Yook J, Yoo TH, Song E, Kim P, Shin E-C, Chung K, Choi K, Choi C (2020). Exosome-based delivery of super-repressor I $\mathrm{KB} \alpha$ relieves sepsis-associated organ damage and mortality. Science Advances 6: eaaz6980. DOI 10.1126/ sciadv.aaz6980.

Emameh RZ, Nosrati H, Eftekhari M, Falak R, Khoshmirsafa M (2020). Expansion of single cell transcriptomics data of SARS-CoV infection in human bronchial epithelial cells to COVID-19. Biological Procedures Online 22: 16. DOI 10.1186/s12575-020-00127-3.

Guo S, Perets N, Betzer O, Ben-Shaul S, Sheinin A, Michaelevski I, Popovtzer R, Offen D, Levenberg S (2019). Intranasal delivery of mesenchymal stem cell derived exosomes loaded with phosphatase and tensin homolog siRNA repairs complete spinal cord injury. ACS Nano 13: 10015-10028. DOI 10.1021/acsnano.9b01892.

Gupta A, Kashte S, Gupta M, Rodriguez HC, Gautam SS, Kadam S (2020). Mesenchymal stem cells and exosome therapy for COVID-19: Current status and future perspective. Human Cell 33: 907-918. DOI 10.1007/s13577-020-00407-w.

Hassanpour M, Rezaie J, Nouri M, Panahi Y (2020). The role of extracellular vesicles in COVID-19 virus infection. Infection, Genetics and Evolution 85: 104422. DOI 10.1016/ j.meegid.2020.104422.

Jabbari N, Akbariazar E, Feqhhi M, Rahbarghazi R, Rezaie J (2020). Breast cancer-derived exosomes: tumor progression and therapeutic agents. Journal of Cellular Physiology 235: 6345-6356. DOI 10.1002/jcp.29668.

Jing H, He X, Zheng J (2018). Exosomes and regenerative medicine: State of the art and perspectives. Translational Research 196: 1-16. DOI 10.1016/j.trsl.2018.01.005.

Kamal MM, Kassem DH (2020). Therapeutic potential of Wharton's jelly mesenchymal stem cells for diabetes: achievements and challenges. Frontiers in Cell and Developmental Biology 8: 16. DOI 10.3389/fcell.2020.00016.

Khare D, Or R, Resnick I, Barkatz C, Almogi-Hazan O, Avni B (2018). Mesenchymal stromal cell-derived exosomes affect mRNA expression and function of B-lymphocytes. Frontiers in Immunology 9: 3053. DOI 10.3389/ fimmu.2018.03053.
Magdy Beshbishy A, Alghamdi S, Onyiche TE, Zahoor M, Rivero-Perez N, Zaragoza-Bastida A, Ghorab MA, Meshaal AK, El-Esawi MA, Hetta HF, Batiha GE (2020). Biogenesis, biologic function and clinical potential of exosomes in different diseases. Applied Sciences 10: 4428. DOI 10.3390/app10134428.

Marino L, Castaldi MA, Rosamilio R, Ragni E, Vitolo R, Fulgione C, Castaldi SG, Serio B, Bianco R, Guida M, Selleri C (2019). Mesenchymal stem cells from the Wharton's jelly of the human umbilical cord: biological properties and therapeutic potential. International Journal of Stem Cells 12: 218-226. DOI $10.15283 /$ ijsc18034.

Parasher A (2020). COVID-19: Current understanding of its pathophysiology, clinical presentation and treatment. Postgraduate Medical Journal, 1-9. DOI 10.1136/ postgradmedj-2020-138577.

Popowski K, Lutz H, Hu S, George A, Dinh PU, Cheng K (2020). Exosome therapeutics for lung regenerative medicine. Journal of Extracellular Vesicles 9: 1785161. DOI 10.1080/ 20013078.2020.1785161.

Sengupta V, Sengupta S, Lazo A, Woods P, Nolan A, Bremer N (2020). Exosomes derived from bone marrow mesenchymal stem cells as treatment for severe COVID-19. Stem Cells and Development 29: 747-754. DOI 10.1089/scd.2020.0080.

Shetty AK (2020). Mesenchymal stem cell infusion shows promise for combating Coronavirus (COVID-19)-induced pneumonia. Aging and Disease 11: 462. DOI 10.14336/AD.2020.0301.

Tay MZ, Poh CM, Rénia L, MacAry PA, Ng LF (2020). The trinity of COVID-19: immunity, inflammation and intervention. Nature Reviews Immunology 20: 363-374. DOI 10.1038/ s41577-020-0311-8.

Wang J, Wu F, Liu C, Dai W, Teng Y, Su W, Kong W, Gao F, Cai L, Hou A, Jiang C (2019). Exosomes released from rabies virusinfected cells may be involved in the infection process. Virologica Sinica 34: 59-65. DOI 10.1007/s12250-019-00087-3.

Xie M, Xiong W, She Z, Wen Z, Abdirahman AS, Wan W, Wen C (2020). Immunoregulatory effects of stem cell-derived extracellular vesicles on immune cells. Frontiers in Immunology 11: 13. DOI 10.3389/fimmu.2020.00013.

Yousef MH, Abdelnaser A (2019). Exosomes: biological couriers with transformative messages. Journal of Biomedicine 4: 14-34. DOI 10.7150/jbm.34611. 